\title{
External jugular venous sampling for Cushing's disease in a patient with hypoplastic inferior petrosal sinuses
}

\author{
Keyan A. Peterson, MS, MBA, ${ }^{1}$ Christofer D. Burnette, RT, ${ }^{2}$ Kyle M. Fargen, MD, MPH, ${ }^{1}$ \\ Patrick A. Brown, MD, ${ }^{2}$ James L. West, MD, ${ }^{1}$ Stephen B. Tatter, MD, PhD, ${ }^{1}$ and \\ Stacey $Q$. Wolfe, MD ${ }^{1}$
}

1Department of Neurosurgery, Wake Forest University School of Medicine; and 'Department of Radiology, Wake Forest Baptist Health, Winston-Salem, North Carolina

\begin{abstract}
The authors report the case of a 30-year-old female patient with suspected Cushing's disease with an anatomical variation of hypoplastic inferior petrosal sinuses and nearly exclusive anterior drainage from the cavernous sinus, who underwent external jugular venous blood sampling with successful disease confirmation and microadenoma localization. The patient presented with signs and symptoms consistent with Cushing's syndrome, but with discordant preliminary diagnostic testing. She underwent attempted bilateral inferior petrosal sinus sampling; however, she had hypoplastic inferior petrosal sinuses bilaterally and predominantly anterior drainage from the cavernous sinus into the external jugular circulation. Given this finding, the decision was made to proceed with external jugular venous access and sampling in addition to internal jugular venous sampling. A positive adrenocorticotropic hormone (ACTH) response to corticotropinreleasing factor was obtained in the right external jugular vein alone, suggesting a right-sided pituitary microadenoma as the cause of her Cushing's disease. The patient subsequently underwent a transsphenoidal hypophysectomy that confirmed the presence of a right-sided ACTH-secreting microadenoma, which was successfully resected. She was hypocortisolemic on discharge and has had no signs of recurrence or relapse at 6 months postoperation.
\end{abstract}

https://thejns.org/doi/abs/10.3171/2019.11.JNS192374

KEYWORDS petrosal sinus sampling; pituitary tumor; venous anatomy; cavernous sinus; cerebral angiography; jugular vein; pituitary surgery

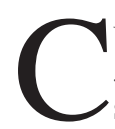
USHING's syndrome (CS) has an incidence of 1 in 500,000 and is characterized by symptoms and signs of hypercortisolemia. ${ }^{9}$ Cushing's disease (CD) is the specific condition of CS caused by a central source, most commonly an adrenocorticotropic hormone (ACTH)-secreting pituitary microadenoma. Noninvasive tests examining blood cortisol, salivary cortisol, and urinary cortisol have variable sensitivity and specificity., 2,6 When CD is suspected, bilateral inferior petrosal sinus sampling (IPSS) is currently the gold standard confirmatory and localizing test. ${ }^{3}$ IPSS involves catheterizing both inferior petrosal sinuses (IPSs) in order to sample blood from each side of the cavernous sinus, the venous drainage of the pituitary gland.

Miller first described the significant variability which can be present at the IPS-internal jugular vein (IJ) junction. ${ }^{7}$ Miller's study of IPS anatomy includes 4 distinct types: types 1 and 2 have normal IPS drainage into the IJ, with type 2 showing a larger contribution from the vertebral plexus; type 3 are venous plexi rather than a distinct IPS; and type 4 (only 5\% of 135 patients) demonstrates anterior cavernous sinus drainage and hypoplastic IPS.? The IPSS test relies on patent IPS and cavernous sinus drainage through the IPS into the IJ vein (Miller types 1 and 2). ${ }^{7}$ When one IPS is absent or atretic there is an absent connection between the IPS and IJ, and as a result the cavernous sinus drains anteriorly because of functionally inadequate IPS (Miller type 4). This anatomy has clinical ramifications for IPSS, as hypoplastic IPSs are a significant source of false negatives and subsequent delays in diagnosis. ${ }^{5} \mathrm{IJ}$ sampling is less sensitive than IPSS when normal posterior cavernous sinus drainage occurs, and even less so if cavernous sinus drainage is directed anteriorly toward the external jugular vein (EJ) in uncommon cases

ABBREVIATIONS $A C T H=$ adrenocorticotropic hormone; $C D=$ Cushing's disease; $C R F=$ corticotropin-releasing factor; $C S=$ Cushing's syndrome; $E J$ = external jugular vein; IJ = internal jugular vein; IPS = inferior petrosal sinus; IPSS = inferior petrosal sinus sampling.

SUBMITTED August 30, 2019. ACCEPTED November 19, 2019.

INCLUDE WHEN CITING Published online January 17, 2020; DOI: 10.3171/2019.11.JNS192374. 

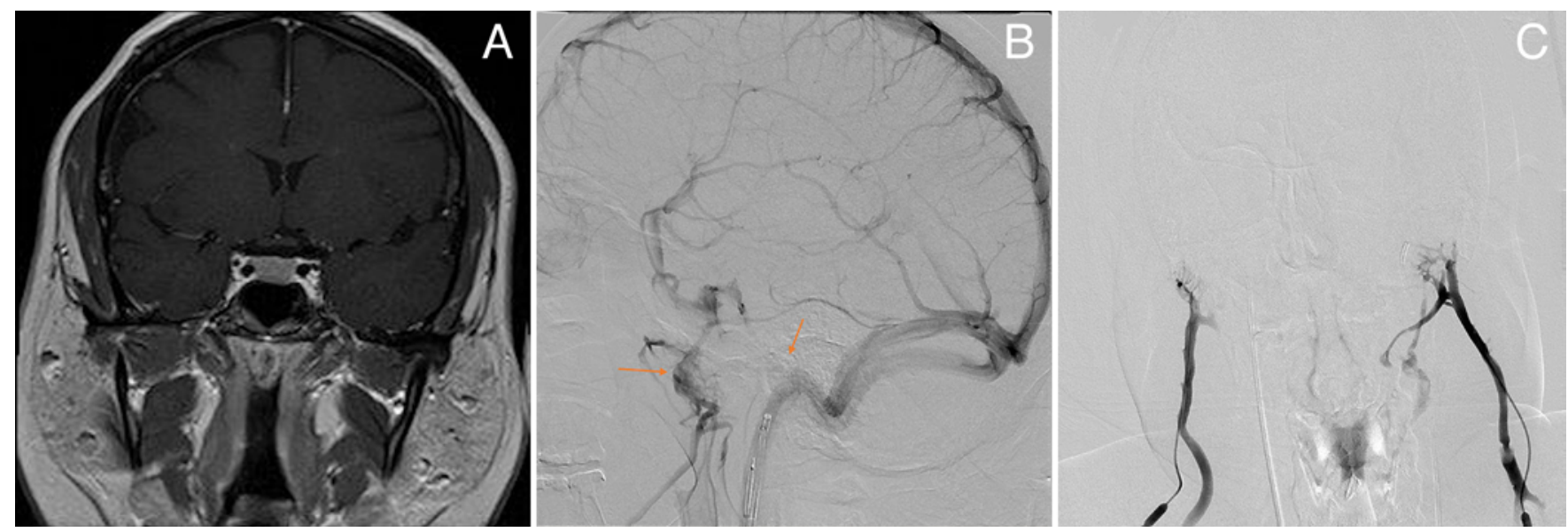

FIG. 1. A: Preoperative coronal dynamic phase-contrast MR image without demonstrable pituitary microadenoma. B: Lateral left carotid venous phase angiogram demonstrating absent IPS-IJ connections (right arrow) and showing extensive anterior cavernous sinus drainage into the EJ and facial vein (left arrow). C: Anteroposterior bilateral EJ venogram demonstrating bilateral EJ access and distal drainage. Figure is available in color online only.

of bilateral IPS hypoplasia. To our knowledge, there have been no reports to date describing successful endocrinologic venous sampling for CD using the EJs.

\section{Case Report}

History

An otherwise healthy 30 -year-old woman presented to her primary care physician with a 40 -pound weight gain and cushingoid appearance over a 6-month period. At presentation her ACTH level was $30.1 \mathrm{pg} / \mathrm{mL}$, with an elevated serum cortisol of $27.1 \mu \mathrm{g} / \mathrm{dL}$. On 1-mg dexamethasone suppression testing her AM serum cortisol was $2.6 \mu \mathrm{g} / \mathrm{dL}$ and with $40 \mathrm{mg}$ it was $5.7 \mu \mathrm{g} / \mathrm{dL}$. At this time, her 24 -hour urine free cortisol was normal at $30 \mu \mathrm{g} / 24$ hours, but discordant with her salivary cortisol, which was elevated at $0.208 \mu \mathrm{g} / \mathrm{dL}$. It should be noted that when the patient first underwent her endocrinology workup, she was taking oral contraceptives, which have been shown in previous studies to be associated with a high rate of false-positive results for hypercortisolism. ${ }^{12}$ The patient underwent dynamic brain MRI with gadolinium-based contrast that demonstrated a hypoenhancing central microcystic sellar lesion consistent with a Rathke's cleft cyst, but the pituitary parenchyma showed no clear pituitary microadenoma to account for her CS (Fig. 1A). Due to these discordant findings, the patient was referred for bilateral IPSS to confirm and lateralize an ACTH pituitary microadenoma.

\section{Intervention and Sampling}

Bilateral IPSS was attempted in the usual fashion., ${ }^{2,4}$ After bilateral common femoral vein access was obtained, guide catheters were navigated into the IJs bilaterally; however, neither IJ-IPS connection could be visualized, and cannulation could not be performed with retrograde venography, a 0.035 -inch wire, an 0.018 -inch microcatheter, or 0.014-inch microwires. Following these failed attempts, femoral arterial access was obtained, and venousphase angiography also failed to demonstrate either IPS-IJ connection. The pituitary venous drainage was carefully assessed and no significant posterior drainage into an IJ could be seen. Interestingly, in this patient the cavernous sinus appeared to drain predominantly via anterior venous outflow into the EJs.

Assessment of the venous phase of the arteriogram in this patient highlighted the almost exclusive utilization of anterior drainage into the EJ and facial vein, left $>$ right (Fig. 1B). Without significant IPS-IJ drainage, and with repeated unsuccessful attempts to cannulate the hypoplastic IPS, the decision was made to cannulate both EJs percutaneously and obtain samples from each EJ. We also performed simultaneous IJ sampling at the expected IPS outlets to maximize sensitivity. Ultrasound guidance was used to directly access each EJ with a 4-Fr micropuncture sheath in a retrograde fashion, and placement was confirmed with venography (Fig. 1C). Following successful placement, venous sampling proceeded according to previously described protocols involving the administration of corticotropin-releasing factor (CRF) and subsequent timed blood collection at 5, 15, and 30 minutes after administration of cosyntropin for assessment of ACTH response. The patient tolerated the procedure well, without any complications. All micropuncture sheaths were then removed under 5 minutes of compression, with excellent hemostasis.

\section{Diagnostic Findings}

At 15 and 30 minutes following CRF administration, the ratio of right EJ to peripheral venous ACTH was 3.14 $(66 \mathrm{pg} / \mathrm{mL})$ and $4.46(125 \mathrm{pg} / \mathrm{mL})$, and the ratio of right to left EJ ACTH was 1.69 and 3.37. Taken together, these results suggested a likely right-sided pituitary microadenoma. These values contrast with peripheral and IJ values measuring $21-39 \mathrm{pg} / \mathrm{mL}$ at all other time points (Table 1).

\section{Surgery and Postoperative Course}

The patient underwent successful transsphenoidal pituitary exploration, with identification and resection of a 
TABLE 1. ACTH levels at baseline and following CRF administration in the EJ, IJ, and peripheral venous blood

\begin{tabular}{lcccc}
\hline \multirow{2}{*}{ Sample Location } & \multicolumn{4}{c}{ ACTH, pg/mL } \\
\cline { 2 - 5 } & Baseline & 5 Mins & 15 Mins & 30 Mins \\
\hline Right IJ & 26 & 11 & 24 & 40 \\
\hline Left IJ & 25 & 11 & 26 & 36 \\
\hline Right EJ & 27 & 22 & 66 & 125 \\
\hline Left EJ & 24 & 13 & 39 & 37 \\
\hline Peripheral & 25 & 14 & 21 & 28 \\
\hline Right EJ/peripheral & 1.08 & 1.57 & 3.14 & 4.46 \\
\hline
\end{tabular}

At 30 minutes after CRF administration, ACTH levels in blood sampled from the right $\mathrm{EJ}$ demonstrated a 4 -fold elevation compared with levels in peripheral venous blood, while ACTH levels in blood from all other sample sites remained stable.

right-sided 0.6-mm microadenoma. Surgical pathology revealed immunopositivity for prolactin, ACTH, and luteinizing hormone (LH) beta, while follicle-stimulating hormone (FSH), thyroid-stimulating hormone (TSH), and human growth hormone (HGH) were negative. A reticulin stain demonstrated loss of the usual reticulin architecture within the nodule, consistent with a corticotroph adenoma. The patient's cortisol level 3 days postoperatively was low at $0.8 \mu \mathrm{g} / \mathrm{dL}$, and she was discharged on a regimen of prednisone to allow time for the preserved normal pituitary to resume adrenocorticotropic functioning. At the 1-month postoperative follow-up, her AM cortisol level was $5.7 \mu \mathrm{g} / \mathrm{dL}$, supporting biochemical remission and surgical cure. Her 6-month postoperative MRI revealed no evidence of recurrent or residual pituitary adenoma, and she is currently scheduled for follow-up MRI with and without contrast in 2 years' time.

\section{Discussion}

At the 150th anniversary of Harvey Cushing's birth, Cushing's disease still carries significant morbidity, and IPSS is essential to confirm the diagnosis when radiographic and laboratory testing is inconclusive or conflicting. The overall success rate for bilateral IPS cannulation for IPSS is approximately $88 \% .^{2}$ Previous reports have addressed unilateral hypoplastic IPS as a cause of falsenegative IPSS and have demonstrated that falsely negative IPSS can result in unintended consequences, including unnecessary procedures and significant delays in diagnosis of up to 30 months. ${ }^{4,5,10}$

Miller's study of IPS anatomy includes 4 distinct types: types 1 and 2 have normal IPS drainage into the IJ, with type 2 showing a larger contribution from the vertebral plexus; type 3 are venous plexi rather than a distinct IPS; and type 4 (only $5 \%$ of 135 patients) demonstrates anterior cavernous sinus drainage and hypoplastic IPS. ${ }^{7}$ In the case we report here, this anatomy led to a novel solution. Retrograde EJ cannulation is a straightforward, low-risk procedure, especially under ultrasound guidance, and is well tolerated by the patient. To our knowledge, this case is the first reported in the literature for which the EJs were accessed to sample the cavernous sinus drainage from the pituitary region to confirm $\mathrm{CD}$ and lateralize a potential microadenoma in a patient with anterior drainage and bilaterally hypoplastic IPS.

Direct cavernous sinus sampling (CSS) and IJ sampling have been reported as alternatives to IPSS for preoperative confirmation and lateralization of microadenomas. While CSS has shown good sensitivity for detecting microadenomas $(82 \%-91 \%),{ }^{1,11}$ this procedure is considerably more invasive than procedures requiring a transsphenodial approach. Radvany et al. demonstrated that IJ sampling has a significantly lower sensitivity (69\%) than does IPSS $(94 \%),{ }^{8}$ likely given the dilution of cavernous sinus blood into overall IJ drainage volumes. In the setting of the anterior cavernous sinus drainage variant, EJ sampling may act in the same manner as IJ sampling and likely suffers the same decreased sensitivity due to large blood volumes from places other than the cavernous sinus. It is important to note that given this dilution the absolute ACTH values will likely be lower than those usually seen in $C D$, as in our patient. Further, even in ideal situations the specificity for lateralization of bilateral IPSS is only 70\%, and though the measurement of the ACTH values allowed for lateralization in this case, EJ sampling cannot be expected to reliably predict lateralization in all patients. ${ }^{13}$

\section{Conclusions}

Anterior drainage of the cavernous sinus may be amenable to endocrinologic sampling using the EJ. While utilization of EJ sampling must be applied selectively, with the aforementioned cautions, in this patient only the EJ results confirmed and lateralized the pituitary microadenoma that caused the patient's CD. If IJ testing had been performed alone in this case, the result would have been negative. Careful assessment of the venous drainage patterns of the cavernous sinus must guide the decision to pursue EJ sampling, and further studies should assess the sensitivity and specificity of this technique.

\section{References}

1. Burkhardt T, Flitsch J, van Leyen P, Sauer N, Aberle J, Grzyska U, et al: Cavernous sinus sampling in patients with Cushing's disease. Neurosurg Focus 38(2):E6, 2015

2. Deipolyi A, Bailin A, Hirsch JA, Walker TG, Oklu R: Bilateral inferior petrosal sinus sampling: experience in 327 patients. J Neurointerv Surg 9:196-199, 2017

3. Deipolyi A, Karaosmanoğlu A, Habito C, Brannan S, Wicky $S$, Hirsch J, et al: The role of bilateral inferior petrosal sinus sampling in the diagnostic evaluation of Cushing syndrome. Diagn Interv Radiol 18:132-138, 2012

4. Deipolyi AR, Hirsch JA, Oklu R: Bilateral inferior petrosal sinus sampling. J Neurointerv Surg 4:215-218, 2012

5. Doppman JL, Chang R, Oldfield EH, Chrousos G, Stratakis CA, Nieman LK, et al: The hypoplastic inferior petrosal sinus: a potential source of false-negative results in petrosal sampling for Cushing's disease. J Clin Endocrinol Metab 84:533-540, 1999

6. England RW, Geer EB, Deipolyi AR: Role of venous sampling in the diagnosis of endocrine disorders. J Clin Med 7:114, 2018

7. Miller DL, Doppman JL, Chang R: Anatomy of the junction of the inferior petrosal sinus and the internal jugular vein. AJNR Am J Neuroradiol 14:1075-1083, 1993

8. Radvany MG, Quinones-Hinojosa A, Gallia GL, Wand GS, 
Salvatori R: Venous sampling for Cushing disease: comparison of internal jugular vein and inferior petrosal sinus sampling. Endocr Pract 22:1057-1061, 2016

9. Ross NS: Epidemiology of Cushing's syndrome and subclinical disease. Endocrinol Metab Clin North Am 23:539-546, 1994

10. Shiu PC, Hanafee WN, Wilson GH, Rand RW: Cavernous sinus venography. Am J Roentgenol Radium Ther Nucl Med 104:57-62, 1968

11. Teramoto A, Yoshida Y, Sanno N, Nemoto S: Cavernous sinus sampling in patients with adrenocorticotrophic hormonedependent Cushing's syndrome with emphasis on inter- and intracavernous adrenocorticotrophic hormone gradients. J Neurosurg 89:762-768, 1998

12. Vastbinder M, Kuindersma M, Mulder AH, Schuijt MP, Mudde AH: The influence of oral contraceptives on overnight $1 \mathrm{mg}$ dexamethasone suppression test. Neth J Med 74:158-161, 2016

13. Wind JJ, Lonser RR, Nieman LK, DeVroom HL, Chang R, Oldfield EH: The lateralization accuracy of inferior petrosal sinus sampling in 501 patients with Cushing's disease. J Clin Endocrinol Metab 98:2285-2293, 2013

\section{Disclosures}

The authors report no conflict of interest concerning the materials or methods used in this study or the findings specified in this paper.

\section{Author Contributions}

Conception and design: West, Tatter, Wolfe. Acquisition of data: Burnette, West, Tatter, Wolfe. Analysis and interpretation of data: Peterson, Fargen, Brown, West, Tatter, Wolfe. Drafting the article: Peterson, Fargen, Brown, West, Wolfe. Critically revising the article: Peterson, Fargen, Brown, West, Tatter, Wolfe. Reviewed submitted version of manuscript: all authors. Approved the final version of the manuscript on behalf of all authors: Peterson. Administrative/technical/material support: Peterson. Study supervision: Wolfe.

\section{Correspondence}

Keyan A. Peterson: Wake Forest University School of Medicine, Winston-Salem, NC.kapeters@wakehealth.edu. 